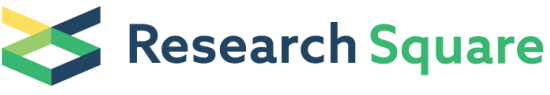 \\ Preprints are preliminary reports that have not undergone peer review. \\ They should not be considered conclusive, used to inform clinical practice, or referenced by the media as validated information.
}

\section{Downregulation of CDK5 Signaling In the Dorsal Striatum Alters Striatal Microcircuits and Perturbs Circadian Behavior in Mice}

Hu Zhou

Beijing Institute of Pharmacology and Toxicology: Academy of Military Medical Sciences Institute of Pharmacology and Toxicology

Jingxin Zhang

Beijing Institute of Pharmacology and Toxicology: Academy of Military Medical Sciences Institute of Pharmacology and Toxicology

\section{Huaxiang Shi}

Beijing Institute of Pharmacology and Toxicology: Academy of Military Medical Sciences Institute of Pharmacology and Toxicology

\section{Pengfei Li}

Beijing Institute of Pharmacology and Toxicology: Academy of Military Medical Sciences Institute of Pharmacology and Toxicology

\section{Xin Sui}

Beijing Institute of Pharmacology and Toxicology: Academy of Military Medical Sciences Institute of Pharmacology and Toxicology

\section{Yongan Wang}

Beijing Institute of Pharmacology and Toxicology: Academy of Military Medical Sciences Institute of Pharmacology and Toxicology

\section{Liyun Wang ( $\square$ lylywang1103@163.com )}

Beijing Institute of Pharmacology and Toxicology: Academy of Military Medical Sciences Institute of Pharmacology and Toxicology https://orcid.org/0000-0002-5253-7091

\section{Research Article}

Keywords: Cyclin-dependent kinase 5 (CDK5), Dorsal striatum, Neuronal projections, Motor activity, Sleepwake behavior

Posted Date: December 30th, 2021

DOI: https://doi.org/10.21203/rs.3.rs-1183888/v1 
License: (c) (i) This work is licensed under a Creative Commons Attribution 4.0 International License. Read Full License 


\section{Abstract}

Dysfunction of striatal dopaminergic circuits has been implicated in motor impairment as well as in Parkinson's disease (PD)-related circadian perturbations that may represent an early prodromal marker of PD. Cyclin-dependent kinase 5 (CDK5) acts negatively on dopamine (DA) signaling in the striatum, suggesting a critical role in circadian and sleep disorders. Here, we used CRISPR/Cas9 gene editing to produce dorsal striatum (DS)-specific knockdown (KD) of the Cdk5 gene in mice (referred to as DS-CDK5$\mathrm{KD}$ mice) to investigate its role in vivo. DS-CDK5-KD mice exhibited deficits in locomotor activity and disturbances in daily rest/activity cycles. Additionally, Golgi staining of neurons in the DS revealed that Cdk5 deletion caused a reduction in dendrite length and functional synapses, which was confirmed by significant downregulation of MAP2, PSD 95 and synapsin I. Correlated with this, DS-CDK5-KD mice displayed reduced phosphorylation of Tau at Thr181. Furthermore, whole-cell patch-clamp recordings of green fluorescent protein (GFP)-tagged neurons in the striatum of DS-CDK5-KD mice revealed a decrease in the frequency of spontaneous inhibitory post-synaptic currents and an alteration of the excitatory/inhibitory synaptic balance. Notably, anterograde labeling showed that CDK5 knockdown in the DS disrupted long-range projections to the secondary motor cortex, dorsal and ventral thalamic nuclei, and the basolateral amygdala, which are involved in the regulation of motor and circadian rhythms in the brain. These findings support a critical role of CDK5 in the DS in maintaining the striatal neural circuitry underlying motor and circadian rhythms related to PD.

\section{Introduction}

Parkinson's disease (PD) is a devastating neurodegenerative disorder characterized pathologically by the loss of dopaminergic neurons in the substantia nigra (SN) pars compacta [1]. The striatum is the main recipient of dopaminergic innervation from the $\mathrm{SN}$ and, accordingly, the impact of its loss on striatal microcircuitry has been extensively studied [2,3]. Dysfunction of striatal dopaminergic neural circuits has been implicated in the pathophysiology of both motor and non-motor symptoms in PD [4, 5]. In addition to the classical motor symptoms, disturbances in daily rest/activity cycles are common non-motor symptoms in PD and may have a substantial impact on the quality of life [6, 7]. It is estimated that rest/activity cycle disturbance in PD emerges when approximately $60 \%$ of nigral neurons have been lost and dopaminergic striatal content is reduced by $80 \%$ [7]. Therefore, restoration of the circadian program in PD has received the attention of the medical and research community [8].

Traditionally, dopamine has been associated with wake-promoting activity. Amphetamines promote wakefulness by enhancing dopamine (DA) release and preventing its reuptake by DA transporters, which further illustrates the wake-promoting effects of DA [9]. Modafinil as a wakefulness inducer has also been linked to dopaminergic activity $[10,11]$. The striatum is the main recipient of dopaminergic innervation from the $\mathrm{SN}$ and is composed primarily of GABAergic medium spiny projection neurons (MSNs) that express $D A$ receptors, $D_{1}$ and $D_{2}[12,13]$. Dopamine and cAMP-regulated phosphoprotein 32 (DARPP-32) is a highly enriched cytosolic protein in MSNs and is considered an important integrator of striatal cellular excitability and synaptic transmission [14]. Dopamine exerts bidirectional control on the phosphorylation 
state of DARPP-32 at Thr34; $D_{1}$ receptors stimulate and $D_{2}$ receptors inhibit this phosphorylation. Additionally, cyclin-dependent kinase 5 (CDK5) phosphorylates DARPP-32 at another site, Thr75, which leads to dephosphorylation of DARPP-32 at Thr34 $[15,16]$. Therefore, CDK5 negatively regulates dopamine signaling in the striatum, indicating a functional role of CDK5 in regulating the rest/activity cycle. In addition, CDK5 is involved in the control of dendritic spine formation and cortical neurotransmission. For example, Cdk5 phosphorylates the NMDA receptor subunit, NR2B, to modulate synaptic transmission [17], and phosphorylates postsynaptic density protein 95 (PSD-95) to promote synaptic PSD-95 clustering and glutamate transmission [18]. These findings indicate that aberrant hypoactivation of CDK5 may contribute to neural circuitry disorders in the human and rodent brain. In mouse models of PD, 1-methyl-4-phenyl-1,2,3,6-tetrahydropyridine (MPTP) treatment results in higher levels of CDK5 and its specific activator, the P25 complex, in dopaminergic neurons, leading to neuronal death [19]. Furthermore, MPTP is toxic to dopaminergic neurons in the substantia nigra, causing irreversible PD symptoms, in which changes to striatal DA levels are major determinants of daily rest/activity cycle perturbations [20-22]. Thus, we predict that high levels of CDK5 will facilitate both DA involvement in the maintenance of wakefulness and inhibition of locomotion in PD, although the exact mechanisms underlying these effects are not completely understood.

Here, we aimed to assess the role of CDK5 in the regulation of circadian rest/activity rhythms using CRISPR/Cas9 gene editing to generate dorsal striatum (DS)-specific Cdk5-deficient mice (referred to as DS-CDK5-KD mice). The DS was chosen because it is a major area of DA involvement in the early stages of motor processing, and DS disturbance may produce the well documented behavioral changes associated with PD [23]. In addition to assessing motor activity, we examined changes in circadian rhythms of sleep-wake behaviors in DS-CDK5-KD mice. Electrophysiological and anterograde labeling studies were used to examine whether CDK5 knockdown impairs cellular excitability and synaptic transmission in the striatal network, which appears to play a key role in the pathophysiology of sleep dysfunction in mice. We aimed to clarify the role of CDK5 in regulating sleep and circadian disturbances associated with PD.

\section{Materials And Methods}

\section{Animals}

Eight-week-old male C57BL/6 mice, initially weighing 18-20 g (Vital River Laboratories, Beijing, China), were group-housed in a controlled environment at $18-22^{\circ} \mathrm{C}$ and $40-60 \%$ humidity, with a $12: 12-\mathrm{h}$ light/dark cycle and access to food and water ad libitum. Enrichment was provided with shredding nestlets. Cages $(32 \times 22 \times 17 \mathrm{~cm}$, eight mice per cage) were changed every week by designated facility staff. All mice were group-housed for 1 week prior to use in an experiment and handled daily to minimize the effects of handling stress. All experiments were performed in accordance with the National Institutes of Health Guide for the Care and Use of Laboratory Animals (NIH Publications No. 80-23) and were approved by the Animal Care and Use Committees of the Beijing Institute of Pharmacology and Toxicology. Efforts were made to minimize the number of animals used for each experiment. 


\section{In vivo knockdown of the Cdk5 gene in mouse DS}

To induce efficient knockdown of mouse CDK5 in the DS, Cdk5-targeting single-guide RNAs (sgRNAs) were designed (Fig. 1A). Lentiviral (LV) vectors (p24 stock solutions ranging between 200 and $300 \mathrm{ng} / \mu \mathrm{L}$, and approximately $2 \times 10^{8}$ transducing units $/ \mathrm{mL}$ ) co-expressing a P2A promoter-driven EGFP and a U6 promoter-driven small guide RNA (sgRNA), either specifically directed against the Cdk5 mRNA (LV-CDK5sgRNA) or a negative control (LV-NC-sgRNA), were produced by GeneChem Co., Ltd (Shanghai, China). The sequence used for targeted editing of Cdk5 was 5'-TCAGCTTCTTGTCACTATGC-3' and the nonspecific negative control sequence was 5'-CGCTTCCGCGGCCCGTTCAA-3'. Standard procedures and validation of LV/Cas9-sgRNA in vivo were reported previously [18]. C57BL/6J mice were anesthetized with pentobarbital sodium [50 mg/kg, intraperitoneal (i.p.), Sigma-Aldrich, St. Louis, MO, USA] and mounted on a stereotaxic apparatus (Zhongshidichuang Science and Technology Development Co., Ltd, Beijing, China). Mice were randomly divided into the wild-type (WT) group, the LV-NC-sgRNA (CDK5-NC) group, and the LV-CDK5-sgRNA (CDK5-KD) group, and the respective lentiviral vectors were bilaterally microinjected into the DS (Fig. 1B). For microinjection, we used the following two bilateral sites in the DS (1.5 $\mu \mathrm{L}$ per site, $3 \mu \mathrm{L}$ total) with stereotaxic coordinates (in $\mathrm{mm}$ ) according to the modified stereotaxic mouse atlas of Paxinos and Franklin [24]: (1) anterior/posterior (A/P) +1.1, medial/lateral (M/L) \pm 1.2 , dorsal/ventral (D/V) -3.2; (2) A/P +0.9, M/L $\pm 2.0, D / V-3.2$. The delivery rate was $0.33 \mu \mathrm{L} / \mathrm{min}$ using a UMP3 microsyringe injector and Micro4 controller (World Precision Instruments, Sarasota, FL, USA). The needles were left in place for 3 min to allow for full delivery of the solutions and then slowly retracted. Next, the scalp was sutured with 7-0 Vicryl suture (ETHICON Co., Ltd, New Jersey, USA).

\section{Experimental design and behavior test}

Mice were screened 3 days before experiment, and mice with abnormal behavior were excluded. Mice were randomly divided into WT, DS-CDK5-NC and DS-CDK5-KD groups, and each group included 24 mice. After finished lentivirus transduction at $14^{\text {th }}$ day, 8 mice from each group were selected for behavioral testing and 4 mice from each group were selected for immunoassay (Fig. 1C). The remaining mice were used for Golgi staining, electrophysiology assays or anterograde labeling. All behavioral experiments were performed at fixed times (9:00-12:00 Am). Each behavioral testing was performed with an interval of $24 \mathrm{~h}$ in the same animals. The testing apparatus was cleaned with a hypochlorous acid solution between subjects. The experimenters were blinded to grouping and drug treatment.

\section{Locomotor activity measurements}

A locomotor activity test was used to assess spontaneous locomotor activity and arousal in mice. The method used was similar to a previously published protocol [25]. Briefly, animals were placed individually in a square arena $(40 \times 40 \times 80 \mathrm{~cm})$ with a Plexiglas floor and walls (Kinder Inc., Poway, CA, USA) and were allowed to move freely. After a $5 \mathrm{~min}$ habituation period, all animal locomotor activities were 
recorded with AnyMaze software (Stoelting Inc., USA), and distance traveled during a 30 min period was recorded and analyzed.

Wheel-running behavioral test

Mice were housed in light and temperature-controlled circadian cabinets (standard mouse circadian cabinet, Actimetrics, Wilmette, IL) within polypropylene cages $(33.2 \times 15 \times 13 \mathrm{~cm})$ containing a metal running wheel (11 cm diameter). Mice were acclimatized to the running wheel for $1 \mathrm{~h}$ prior to assessment. Locomotor activity rhythms were monitored with a ClockLab data collection system (Version 3.603, Actimetrics, Wilmette, IL) through the number of electrical closures triggered by wheel rotations. Cage changes were scheduled at 24-h intervals. Wheel-running activity was collected over a period of $24 \mathrm{~h}$ and analyzed using ClockLab Analysis software (Actimetrics Software).

\section{Golgi staining and dendritic spine measurement}

Golgi-Cox staining was performed using a Rapid Golgistain Kit (FD Neuro Technologies, Ellicott City, MD, USA) following the manufacturer's instructions. Briefly, brains were quickly removed and rinsed, and incubated in a mix of solution A/B for 14 days in the dark at room temperature. Then, solution $A / B$ was changed to solution $C$ for 3 days. Coronal sections of the DS $(200 \mu \mathrm{m}$ thick) were cut (ranging from 0.7$1.2 \mathrm{~mm}$ anterior to bregma; two sections per animal) on a freezing microtome (Leica, Wetzlar, Germany) and mounted onto gelatinized slides. After sections were dried in the dark, they were reacted in solutions $D$ and $E$ for 10 minutes, and dehydrated sequentially in 50, 75, 95 and 100\% ethanol. Finally, sections were cleared in xylene and cover slipped with resinous mounting medium.

For dendritic spine measurement, the DS region was identified at low power (100 × magnification), and MSNs were traced at $250 \times$ (final magnification) using the camera lucida technique on an Olympus light microscope (Model BX51) equipped with a drawing attachment. MSNs were traced using the 8-bit ImageJ plugin, Neuron J. Dendrite length and branching were measured using a Sholl analysis of ring intersections. A series of concentric rings at $20 \mu \mathrm{m}$ increments printed on a transparency was centered over the soma. The total number of intersections between each ring and dendritic branches was counted and converted to estimates of dendrite length as a function of distance from the soma (i.e., for each 20 $\mu \mathrm{m}$ segment) and overall dendrite length. Spine density was measured manually in the stacks using the ImageJ Plugin, Cell Counter. Three to five dendrite segments per slice, with each dendrite segment ranging from 20 to $30 \mu \mathrm{m}$ in length, were used for spine density analysis. Spines were marked in the appropriate focal plane, preventing any double counting of spines.

\section{Immunohistochemistry}

After the last behavioral test, mice were euthanized and striatum sections were prepared for immunohistochemistry as previously described (Huang et al., 2016). Briefly, striatal sections $(30 \mu \mathrm{m})$ were cut with a vibratome (VT1000 S, Leica) and collected in PBS as free-floating sections. Sections were 
rinsed three times in PBS and permeabilized and blocked in PBS containing 0.3\% Triton X-100 and 5\% normal goat serum (Pierce Biotechnology, Rockford, IL, USA) for $1 \mathrm{~h}$ at room temperature. Sections were then washed in PBS and incubated overnight at $4^{\circ} \mathrm{C}$ with anti-CDK5 antibody (1:400; Cat\# ab40773, Abcam, Cambridge, England), which was detected with an anti-rabbit Alexa Fluor 594 secondary antibody (1:200; Ex: 590 nm, Em: 617 nm; Jackson ImmunoResearch, West Grove, PA, USA). To identify nuclei, sections were counterstained in mounting medium containing DAPI (Sigma-Aldrich). Finally, sections were mounted and examined using a fluorescence microscope (Zeiss, Oberkochen, Germany or Leica, Germany).

\section{Western blotting}

Western blot assays were used to measure protein levels. After the last behavioral test, mice were euthanized with pentobarbital sodium salt (50 mg/kg, i.p.). The striatum was dissected and homogenized in RIPA lysis buffer [10 mM PBS, pH 7.4, containing 1\% NP-40, 0.5\% sodium deoxycholate, $0.1 \%$ SDS, and complete protease inhibitor cocktail (Roche, Basel, Switzerland)]. Tissue dissection was performed in the same time window for all groups. For western blotting, solubilized proteins ( $50 \mu \mathrm{g}$ per sample) were resolved on SDS-PAGE gels and transferred onto polyvinylidene fluoride membranes (Millipore, Bedford, MA, USA). The membranes were blocked with $5 \%$ non-fat milk diluted in TBST at room temperature for 1 $h$, and then incubated overnight at $4{ }^{\circ} \mathrm{C}$ with the following primary antibodies: anti-CDK5 mouse monoclonal antibody (1:1,000; Cat\# ab40773, Abcam), anti-MAP2 rabbit polyclonal antibody (1:1,000; Cat\# ab183830, Abcam), anti-Synapsin1 mouse monoclonal antibody (1:1,000; Cat\# MAN894, Millipore), anti-PSD95 rabbit polyclonal antibody (1:100 dilution, Cat\# ab12093, Abcam), anti-Phospho-Tau (Ser202) rabbit polyclonal antibody (1:1,000; Cat\# 11834s, Cell Signaling Technology, MA, USA), anti-Phospho-Tau (Thr181) rabbit polyclonal antibody (1:1,000; Cat\# 12885s, Cell Signaling Technology), anti-Tau mouse monoclonal antibody (1:1,000; Cat\# MAB3420, Millipore), and anti- $\beta$-actin mouse monoclonal antibody (1:1,000; AF0003, Beyotime). The membranes were then washed with TBST and incubated with the appropriate peroxidase-conjugated secondary goat anti-rabbit/mouse IgG (1:2,000; Cat\# AB-2301/ZB2305, ZSGB-Bio, Beijing, China) for $1 \mathrm{~h}$ at room temperature. An ECL detection kit (Thermo Fisher Scientific, Waltham, MA) was used and immunoreactive bands were quantified on an imaging system (ProteinSimple, San Jose, CA, USA). $\beta$-actin was used as the loading control.

\section{Anterograde labeling}

To characterize the dopaminergic projections to different brain regions, AAV-hSyn-mCherry-IRES-WGA-Cre was packaged (SunBio Biomedical Technology Co. Ltd., Shanghai). On day 14 after Cdk5-sgRNA injection, the AAV-hSyn-mCherry-IRES-WGA-Cre was bilaterally microinjected into the center of the original virus injection points (coordinates: A/P $+1.0 \mathrm{~mm}, \mathrm{M} / \mathrm{L}+1.8 \mathrm{~mm}, \mathrm{D} / \mathrm{V}-3.2 \mathrm{~mm}$ ). Animals were anesthetized throughout surgery with pentobarbital sodium (50 mg/kg, i.p.). Following craniotomy, a microsyringe was lowered into the brain region of interest, and $500 \mathrm{~nL}$ of AAV-hSyn-mCherry-IRES-WGACre was injected over a 10 min period [26]. Then, 30 days later, mice were deeply anesthetized and 
immediately perfused transcardially with normal saline followed by $4 \%$ paraformaldehyde (PFA)/phosphate buffer. Brains were removed and postfixed overnight in the same solution, cryoprotected by immersion in $30 \%$ sucrose, and then frozen in dry ice-cooled methyl butane. Serial coronal cryostat sections $(40 \mu \mathrm{m})$ through the whole brain were cut with a vibratome (VT1000 S, Leica), rinsed in PBS, counterstained with the nuclear dye, 4囚,6-diamidino-2-phenylindole (DAPI) (0.2 mL; Zsbio, Beijing, China), and mounted on slides. Images were captured with a microscope slide scanner (Pannoramic SCAN II, 3DHistech, Ltd., Budapest, Hungary). Coronal sections of the cortex, striatum, thalamus and basolateral amygdala were confirmed post hoc in the microscope slide scanner. CaseViewer 2.3 software (3DHistech, Ltd.) was used for image observation and analysis.

\section{Electrophysiology}

Mice were anesthetized with pentobarbital sodium (50 mg/kg, i.p.), and brains were removed. Striatal slices $(300 \mu \mathrm{m})$ were cut in ice-cold cutting solution [(in mM) $124 \mathrm{NaCl}, 2.8 \mathrm{KCl}, 1.25 \mathrm{NaH}_{2} \mathrm{PO}_{4}, 2 \mathrm{CaCl}_{2}$, $1.25 \mathrm{MgSO}_{4}, 26 \mathrm{NaHCO}_{3}, 10$ glucose; $\mathrm{pH} 7.3$; bubbled with $95 \% \mathrm{O}_{2} / 5 \% \mathrm{CO}_{2}$ ] using a vibrating tissue slicer (MA752, Campden Instruments, Loughborough, UK). Coronal slices were submerged for $30 \mathrm{~min}$ at $32^{\circ} \mathrm{C}$ in artificial cerebrospinal fluid (ACSF) [(in mM) $126 \mathrm{NaCl}, 2.5 \mathrm{KCl}, 26.2 \mathrm{NaHCO}_{3}, 1.25 \mathrm{NaH}_{2} \mathrm{PO}_{4}, 2 \mathrm{CaCl}_{2}, 1.5$ $\mathrm{MgSO}_{4}, 10$ D-glucose, 5 sodium ascorbate; $\mathrm{pH} 7.3$; bubbled with $95 \% \mathrm{O}_{2} / 5 \% \mathrm{CO}_{2}$ ] at 295-300 mOsm/L. All reagents were from Sigma-Aldrich (St. Louis, MO, USA) unless otherwise indicated. Following this recovery period, slices were transferred to room-temperature ACSF until recording.

Patch pipets were prepared from borosilicate glass (Sutter Instrument Company, Novato, CA, USA) using a P-97 Flaming/Brown micropipette puller (Sutter Instrument Company) and had a resistance of 6-8 M $\Omega$ when filled with the following intracellular solution [(in mM) $130 \mathrm{CsCl}, 10 \mathrm{NaCl}, 0.25 \mathrm{CaCl}_{2}, 2 \mathrm{MgCl}_{2}, 5$ EGTA, 10 HEPES, 10 glucose, $2 \mathrm{Mg}$-ATP, $0.3 \mathrm{Na}_{2}$-GTP]. The pH of the pipet solution was adjusted to 7.3 with $1 \mathrm{mM} \mathrm{CsOH}$, and osmolarity was adjusted to 285-290 mOsm/L. A low-power objective (4×) was used to identify the DS region, and a 40× water immersion objective (NIR Apo, Nikon, Japan) coupled to an infrared differential interference contrast (IR-DIC) microscope with a fluorescence system and a CCD camera was used to visually identify, patch, monitor and record CDK5-KD neurons in the DS. MSNs were identified according to previously determined membrane characteristics and firing properties [27]. Recording in normal current-clamp or voltage-clamp modes was performed with a Digidata 1440A digitizer, an Axon 200B amplifier, and Clampex 10.2 software (all from Molecular Devices, San Jose, CA, USA) at room temperature. Fast and slow capacitance compensation was performed after tight-seal ( $>1$ $\mathrm{G} \Omega$ ) formation. During whole-cell recordings, series resistance was monitored and compensated (80$90 \%$ ) periodically. When the series resistance of a neuron was above $50 \mathrm{G} \Omega$ or changed by more than $25 \%$, it was excluded from further analysis. Data were filtered at $2 \mathrm{kHz}$ and acquired at a sampling rate of $10 \mathrm{kHz}$. Access resistance and leak currents were monitored, and recordings were rejected if these parameters changed significantly during data acquisition. 
For miniature excitatory postsynaptic current (mEPSC) recordings, the oxygenated ACSF contained the GABA receptor antagonist, (+)-bicuculline (10 $\mu \mathrm{M}$; Sigma-Aldrich), and the voltage-gated sodium channel blocker, tetrodotoxin (TTX) ( $1 \mu \mathrm{M}$; Abcam), to abolish inhibitory postsynaptic current (IPSC) events and action potentials. For spontaneous IPSC (sIPSC) recordings, the oxygenated ACSF contained the competitive NMDA receptor antagonists, 6-cyano-7-nitroquinoxaline-2, 3-dione (CNQX) (20 $\mu$ M; SigmaAldrich) and L-(+)-2-amino-5-phosphonopentanoic acid (L-AP5) (50 $\mu \mathrm{M}$; Tocris, Ellisville, MO, USA), as well as TTX $(1 \mu \mathrm{M})$ to abolish excitatory postsynaptic current events and action potentials. The sections were superfused with the oxygenated ACSF solutions at a rate of $1.2 \mathrm{~mL} / \mathrm{min}$. Spontaneous activity was recorded $5 \mathrm{~min}$ after whole-cell mode was obtained, for a period of at least $3 \mathrm{~min}$. Data were analyzed using Clampfit 10.2 (Molecular Devices), OriginPro 2018 (Origin Lab, Washington, MA, USA) and/or GraphPad Prism 7 (GraphPad, San Diego, CA, USA).

\section{Statistical analysis}

GraphPad Prism 8.0 software (GraphPad Software, Inc, La Jolla, CA, USA) was used for behavior, western blot, fluorescence image and Golgi staining analysis. Clampex 10.2 software (Molecular Devices, Union City, CA, USA) was used for electrophysiology analysis, and the Kolmogorov-Smirnov test was used to compare the cumulative distributions of frequency and amplitude of groups. Differences between groups were determined by one-way analysis of variance (ANOVA) followed by Tukey's multiple comparisons test. All results are expressed as the mean \pm standard error of the mean (SEM). The number of samples/subjects per experiment is noted in the corresponding figure legend. A $p$-value $<0.05$ was considered statistically significant.

\section{Results}

\section{Motor impairment after lentiviral delivery of $C d k 5$ sgRNA into the DS of mice}

We generated DS-CDK5-KD mice using the CRISPR-Cas9 system, and Cdk5 sgRNA was targeted to the DS by microinjection of a lentiviral vector. Immunofluorescence studies revealed abundant CDK5 staining (red) in the DS in mice transfected with LV/Cas9-NC-sgRNA, while CDK5 staining (red) was greatly reduced in virus-transfected cells (green) in DS-CDK5-KD mice (Fig. 1D). Similar results were confirmed by western blotting. CDK5 expression in the DS of mice injected with LV/Cas9-NC-sgRNA did not change significantly, whereas CDK5 expression was significantly decreased in mice injected with LV-CDK5-sgRNA [Fig. 1E; NC-sgRNA, 98.57\% $\pm 16.89 \%$ vs CDK5-sgRNA, 65.36\% $\pm 15.69 \%, F(2,9)=4.99, \mathrm{P}=0.0347$ ]. Taken together, the immunostaining and western blot analyses showed that LV/Cas9-sgRNA efficiently knocked down CDK5 in DS neurons by day 14 after injection.

We then examined whether the reduction of CDK5 expression in the DS caused changes in behavioral performance. Locomotor activity was continuously monitored throughout day 14 after LV/Cas9-sgRNA 
injection. The distance plot of DS-CDK5-KD mice in 5-min blocks revealed severe spontaneous general activity abnormalities (Fig. 2A). Statistical analysis showed that both the total distance traveled [Fig. 2B; WT, $21965.75 \pm 1,827.91 v s$ DS-CDK5-KD, $13465.04 \pm 1265.40 ; \mathrm{F}(2,21)=8.54, p=0.0019]$ and the total velocity [Fig. 2C; WT, $5.49 \pm 0.34$ vs DS-CDK5-KD, $3.74 \pm 0.35 ; \mathrm{F}(2,21)=6.73, p=0.006$ ] were significantly reduced for DS- CDK5-KD mice. In addition, the activity heatmap revealed that DS-CDK5-KD mice preferred to travel in the peripheral area more than the central area of the arena (Fig. 2D). In line with this, statistical analysis showed that the central distance traveled was significantly decreased in DS-Cdk5-KD mice [Fig. 2E; WT, $1524.08 \pm 157.52$ vs DS-CDK5-KD, $991.25 \pm 146.93, \mathrm{~F}(2,21)=6.24, p=0.01]$. In addition, DS-CDK5-KD mice spent more time nest building like Fig $2 \mathrm{~F}$ and curled up during the test period [Fig. 2G; WT, $1,037 \pm 62.01$ vs DS- CDK5-KD, 1,589.00 $\pm 133.19, \mathrm{~F}(2,21)=8.64, p=0.0018]$. This could simply be a matter of comfort as animals seek warmth to prepare for sleeping. In contrast, both WT and DS-CDK5-NC mice exhibited normal free-walking activity. In line with the immunostaining and western blot analyses showing efficient knockdown of CDK5 in the DS, the altered behavior indicated that knockdown of CDK5 in the DS affects the DS neural network that controls motor behavior.

\section{The circadian rhythm of wheel-running activity is altered in DS-CDK5- KD mice}

To examine the DS-specific CDK5-driven 24-h night/day rhythms of locomotor activity (free-running), wheel-running activity was continuously monitored during the normal night-day cycle (12/12-h). As shown as Fig. 3A, the WT and DS- CDK5-NC mice sustained normal rhythms of sleep-wake behavior with free movement and foraging during the night (12-h night period) and lower overall activity during the daytime (12-h day period). However, the free-running period of DS- CDK5-KD mice at night was significantly shorter than that of WT and DS-CDK5-NC mice. The spontaneous locomotor activity of DSCDK5-KD mice was only $\sim 20 \%$ of the total activity of control littermates. The activity counts of DS-CDK5KD mice were significantly reduced [Fig. 3B; WT, 3,549.54 \pm 154.20 vs DS-CDK5-KD, 594.77 $\pm 64.47, \mathrm{~F}(2$, $21)=38.402, p<0.001]$ at night. However, no significant differences were observed in activity duration between WT, DS-CDK5-NC, and DS-CDK5-KD mice in 12-h night/12-h day free running (Fig. 3C,). Thus, we calculated the ratio of the dark (a) to light $(\rho)$ phase counts in the night-day cycle. No significant differences in $\alpha / \rho$ ratio were observed between WT and DS- CDK5-NC mice. The $\alpha / \rho$ ratio was significantly smaller in DS-CDK5-KD mice than in WT mice [Fig. 3D; WT, 20.34\% $\pm 2.92 \%$ vs DS-CDK5-KD, $6.78 \% \pm 1.01 \%, \mathrm{~F}(2,21)=7.64, p=0.003]$. Corresponding with previous studies, the $\alpha / \rho$ is positively correlated with the circadian period, and abnormal $\alpha / \rho$ indicates a disturbed circadian rhythm. Next, bivariate correlations with behavioral findings indicated that the $\alpha / \rho$ ratio decrease in wheel running activity significantly correlated with other behaviors, such as curling up in the locomotor activity test [Fig. $\left.3 E, r(8)=0.74, p<0.001 ; R^{2}=0.58\right]$. This reduction in overall $a / \rho$ ratio activity in DS-CDK5-KD mice indicates that CDK5 is involved in circadian rhythm behavior in mice. Consistently, bivariate correlations indicated that changes in circadian rhythm may contribute to impaired locomotor activity. 


\section{CDK5 deficiency causes morphological alterations of MSN dendrites and spines in the DS}

CDK5 is required for radial neuronal dendrite and spine maintenance [28] and dysregulation of CDK5 dramatically affects striatal-dependent brain function. Here, we show the stratification of apical MSN morphology in the striatum of WT and DS-CDK5-NC mice and significant changes in neuronal morphology in DS-CDK5-KD mice as determined by Golgi staining (Fig. 4A). Dendrite lengths in WT (746.3 $\pm 40.81 \mu \mathrm{m}, n=20 \mathrm{MSNs})$ and DS-CDK5-NC (725.0 $\pm 62.49 \mu \mathrm{m}, n=12 \mathrm{MSNs})$ mice were not significantly different from each other. However, in DS-CDK5-KD mice, dendrite length $(142.8 \pm 15.00 \mu \mathrm{m}, n=12$ MSNs) was significantly reduced [Fig. 4B; F $(2,25)=48.91, p<0.0001]$. By concentric circle (Sholl's) analysis, we found a significant decrease in dendritic branching in DS-CDK5-KD mice (Fig. 4C). The impairment of dendritic branching occurred proximal (rings 3-8), then distal to the soma (rings 9-20), indicating significantly decreased MSN neurite arborization in DS-CDK5-KD mice. Meanwhile, total dendritic branching was significantly reduced in DS-CDK5-KD mice (56.50 $\pm 7.68, n=12$ MSNs) compared with that in WT $(108.5 \pm 6.98, \mathrm{n}=20 \mathrm{MSNs})$ and DS-CDK5-NC (86.50 $\pm 8.66, \mathrm{n}=20 \mathrm{MSNs})$ mice [Fig. 4D; $F(2,15)=11.18, p=0.0011]$. CDK5 knock down in the DS also impaired MSN spine generation and caused a significant decrease in dendritic spine density. Representative sections of dendritic spines are shown for each group in Fig. 4E. The dendritic spine density per $20 \mu \mathrm{m}$ was significantly reduced in DSCDK5-KD mice (5.23 $\pm 0.52, n=15$ segments) compared with that in WT (12.61 $\pm 0.79, n=16$ segments) and DS-CDK5-NC (11.21 $\pm 0.77, n=16$ segments) mice [Fig. 4F; F $(2,48)=27.37, p<0.0001]$.

Western blot analysis determined the levels of synaptic and spine formation proteins (Fig. 5A), including MAP2, synapsin 1 (presynaptic proteins), and PSD95 (postsynaptic protein). Statistical analysis revealed that DS-CDK5-KD mice had significantly decreased levels of synapsin 1 [Fig. $5 \mathrm{~B}$; WT, $103.0 \% \pm 5.19 \% \mathrm{vs}$ DS-CDK5-KD, 70.77\% $\pm 3.73 \% ; \mathrm{F}(2,9)=14.86, p=0.0014$ ], MAP2 [Fig. 5B; WT, $102.4 \% \pm 2.55 \%$ vs DSCDK5-KD, 78.25\% $\pm 3.05 ; F(2,9)=20.26, p=0.0005$ ] and PSD95 [Fig. 5B; WT, $95.26 \% \pm 5.07 \%$ vs DSCDK5-KD, 61.59\% $\pm 10.20 \% ; \mathrm{F}(2,9)=6.74, p=0.0162]$. Next, we examined Tau protein and its phosphorylation sites, Thr181 and Ser202 (Fig. 5C), which are closely related to synapse reconstruction and neuronal morphology maintenance. Compared with WT mice, total Tau protein levels were significantly reduced in DS-CDK5-KD mice [Fig. 5D; WT,97.72\% $\pm 3.68 \%$ vs DS-CDK5-KD, 73.08\% $\pm 2.16 \%$; $F(2,9)=20.92, p=0.0004]$. Interestingly, there were no changes in the phosphorylation of Tau at Ser202 in DS-CDK5-KD mice [Fig. 5D; WT, 98.94\% $\pm 3.07 \%$ vs DS-CDK5-KD, 87.60\% $\pm 1.38 \%$; $F(2,9)=3.06, p=$ 0.097]. However, we found a significant decrease in phosphorylation of Tau1 at Thr181 in DS-CDK5-KD mice compared with WT mice, indicating a reduction in the phosphorylation activity of CDK5 at Thr181 [Fig. 5D; WT, 108.2\% $\pm 3.78 \%$ vs DS-CD K5-KD, 87.41\% $\pm 2.85 \% ; F(2,6)=14.37, p=0.0052]$. These data indicate that downregulation of CDK5 in the DS perturbs dendrite branching and spine formation, as well as the phosphorylation of Tau1 at Thr181 and that CDK5, therefore, plays a key role in synaptic transmission. Together, these results demonstrate that deletion of CDK5 causes morphological disruption and biochemical changes in DS neurons. 


\section{CDK5 deficiency affects inhibitory synaptic transmission in the DS}

Deletion of Cdk5 dramatically affects the morphology of MSNs in the DS, which may in turn affect the neuronal signal transduction that underlies behavior. We therefore recorded mEPSCs and sIPSCs using the whole-cell techniques. Electrode placement and action potential properties recorded from MSNs in brain slices are shown in Fig. 6A. The mEPSCs reflect the presynaptic release of neurotransmitter from vesicles (Fig. 6B). In DS-Cdk5-KD mice, there was no change in the frequency of mEPSCs [Fig. 6C; WT: $7.48 \pm 0.69 \mathrm{~Hz}$ vs DS-Cdk5-KD: $7.80 \pm 0.70 \mathrm{~Hz}, \mathrm{~F}(2,33)=0.20, p=0.82$ ], or in the average amplitude [Fig. 6 C; WT: $27.32 \pm 1.54$ pA vs DS-Cdk5-KD: $26.53 \pm 2.40$ pA, F(2, 33) $=1.44, p=0.25]$. mEPSCs recorded in the presence of TTX showed that the amplitude and frequency did not change. Therefore, we recorded sIPSCs without TTX, and studied the impact of Cdk5 deletion on inhibitory synapses and changes to inhibition in the loop (Fig. 6D). sIPSC amplitude was unaffected in DS-Cdk5-KD mice [Fig. 6E; WT: $32.17 \pm$ 2. 39 pA vs DS-Cdk5-KD: $29.17 \pm 3.26$ pA, $F(2,33)=0.51, p=0.61]$. However, the frequency of sIPSCs was reduced in DS-Cdk5-KD mice [Fig. 6E; WT: $6.22 \pm 0.82 \mathrm{~Hz}$ vs DS-Cdk5-KD: $3.41 \pm 0.48 \mathrm{~Hz}$; F(2, 33) = 6.59, $p=0.0039]$. These findings indicate that dysregulation of Cdk5 in the striatum may alter synaptic transmission within the DS in a manner that is consistent with the morphological and behavioral deficits.

\section{CDK5 deficiency in the DS reduces long-range projections to the secondary motor cortex, thalamic nuclei and basolateral amygdala}

To examine whether Cdk5 knockdown in the DS affects striatal-dependent brain function, we employed anterograde tracing with AAV-hSyn-mCherry to label fiber tracts that project from the DS (Fig. 7A). This sensitive neuroanatomical tract tracing technique can be used to visualize neuronal projections, including dendritic arbors [29]. All injections were centered in the DS [coordinates: $A / P+1.0 \mathrm{~mm}, \mathrm{M} / \mathrm{L}+1.8 \mathrm{~mm}, \mathrm{D} / \mathrm{V}$ -3.2 mm from bregma (Paxinos and Watson, 1986; Franklin and Paxinos, 1997)] and were visualized using fluorescence microscopy to check for injection accuracy. Striatal sections obtained after anterograde AAV injection showed that most striatal neurons expressed the mCherry tracer in the DS region (Fig. 7B). Four weeks after AAV-hSyn-mCherry microinjection, whole brain sections were used to trace long-range axonal connections from the DS to the secondary motor cortex (M2), dorsal thalamic nuclei, and the basolateral amygdala (BLA), regions that are closely associated with regulating motor function and circadian rhythm. Fig. 7C, D illustrates the pattern of anterograde labeling observed in the M2, thalamus and BLA in WT, DS-Cdk5-NC and DS-Cdk5-KD mice. In WT and DS-Cdk5-NC mice, the majority of anterogradely labeled cells were located in the M2, thalamic nuclei and BLA, which revealed close contact of all three brain regions with the DS. By contrast, the viral tracers were unevenly and weakly present in the M2, thalamus and BLA of DS-Cdk5-KD mice. Bar graphs of mCherry fluorescence in the M2, thalamus and BLA show that fluorescence intensities in all three brain areas were significantly reduced in DS-Cdk5-KD mice compared with WT mice [mCherry fluorescence in the M2 (Fig. 7C): WT, $100.0 \% \pm 6.49 \%$ vs DS-Cdk5-KD, 33.91\% $\pm 1.21 \% ; F(2,6)=40.68, p=0.003$; mCherry fluorescence in the thalamus (Fig. 7D): WT,100.0\% $\pm 2.78 \%$ vs DS-Cdk5-KD,52.97\% $\pm 1.40 ; F(2,9)=40.81, p=0.0003$; mCherry fluorescence in the BLA (Fig. 7E): WT, $100.1 \% \pm 8.27 \%$ vs DS-Cdk5-KD, 41.72\% $\pm 4.37 \%$; F $(2,6)=18.43, p$ 
$=0.0027]$. These findings indicate that Cdk5 is important for neural connectivity between the DS and the $M 2$, thalamus and BLA. The DS is the main integration station of the basal ganglia, and CDK5 may have a critical role in maintaining the neural circuits associated with motor function and circadian rhythms in the DS.

\section{Discussion}

Striatal dopamine signaling is associated with circadian modulations in the mammalian brain. CDK5 regulation of dopamine neurotransmission in the striatum has been previously evaluated, but the role of CDK5 in circadian regulation is unknown. We therefore used a lentiviral-based CRISPR/Cas 9 system to efficiently knockdown the Cdk5 gene in the DS of mice. The DS-CDK5-KD mice exhibited behavioral deficits in locomotor activity and disturbed daily rest/activity cycles, along with dendrite and spine morphological abnormities and impaired basal GABA-mediated sIPSCs in the DS. Furthermore, CDK5 deficiency reduced long range connections from the $D S$ to the $M 2$, thalamus and BLA. These findings provide insight into the involvement of striatal CDK5 in circadian modulations.

The study of sleep and alertness in neurodegenerative disorders, including PD, is very challenging. In our study, using CRISPR/Cas9-mediated gene editing, we efficiently achieved selective knockout of the Cdk5 gene in the DS of mice (Fig. 1C, D). Fourteen days after viral vector injection, WT and DS-CDK5-NC mice displayed normal locomotor behaviors during the rest/activity cycle. In contrast, DS-CDK5-KD mice exhibited marked reductions in total distance traveled and average moving speed. Moreover, DS-CDK5-KD mice adopted a particular posture (curling up) that coincided with the time at which they were most likely to sleep during the test period (Fig. 2D-F). In addition, we examined the effect of DS-specific CDK5 knockdown on circadian rhythms of rest/activity behaviors using the running wheel. The circadian system generates a 24-h rhythm of sleep-wake behavior. Control littermates exhibited a circadian rhythm of free-running locomotion, whereas locomotor rhythms in DS-CDK5-KD mice were severely disrupted during the 12-h night period (Fig. 3B, C), with the mice displaying poorly consolidated subjective nighttime activity. The loss of behavioral rhythmicity indicates that striatal CDK5 is largely associated with wakefulness.

CDK5 is an important Ser/Thr protein kinase that participates in actin-binding, synaptic morphology maintenance and postsynaptic organization [30]. CDK5 also regulates the trafficking of synaptic vesicles and neurotransmitter release and contributes to homeostatic scaling [31, 32]. Consistently, in DS-CDK5KD mice, we found changes in dendritic branching and spine formation, as well as altered Cdk5dependent phosphorylation of Tau at Thr181 in the DS region. Proper spine density and morphology, as well as a balance between synaptic excitation and inhibition ( $E / I$ balance) is widely regarded to be essential for sleep-wake rhythms [33]. Furthermore, most E/I synapses in the brain are on dendritic spines, which are small protrusions on dendritic shafts that are important for synaptic plasticity $[34,35]$. Wholecell recording in DS-CDK5-KD mice revealed a specific decrease in GABA-mediated sIPSCs, while no change was observed in mEPSCs. The GABAergic system, particularly in MSNs, is the major inhibitory neurotransmitter system that underpins E/I balance in the central nervous system. Dysfunction of 
GABAergic neurotransmission has been implicated in the pathogenesis of numerous behavioral conditions [36-39]. Therefore, CDK5 may perturb cytoskeletal assembly and spine density, thereby affecting GABAergic synaptic E/I balance in the striatum and reducing the inhibitory output of MSNs (Fig. 6B-D), in turn resulting in altered sleep-wake rhythms.

Brain functions are mediated by multiple neuronal activities involving highly elaborate and complex synaptic connections. Neurons need to transport organelles, proteins and lipids from the soma to the axon and dendrites and back again to maintain a normal functional state [40,41]. Microtubules act as conduits for both anterograde and retrograde transport of molecules $[42,43]$. We found here that total Tau expression and the phosphorylation of Tau at Thr181 was significantly decreased in DS-CDK5-KD mice (Fig. 5C, D). Tau is highly enriched in neurons and was originally identified by its ability to bind to and stabilize microtubules. The equilibrium between Tau phosphorylation and dephosphorylation modulates the stability of the cytoskeleton and synaptic morphology in the normal brain. Consistent with this, we found that CDK5 deletion perturbed the anterograde trafficking of AAV from the DS to the M2, thalamic nuclei and BLA. Nighttime sleep disturbances are common in PD, affecting up to $90 \%$ of PD patients [6]. The M2, thalamic nuclei and BLA are closely related with sleep-wake rhythms. The M2 is important for processing and integrating sensorimotor cues, and is involved in motor planning [44]. The thalamus is a critical node that integrates input and output in the central nervous system, and striatalthalamic connections are the foundation of many higher brain functions $[45,46]$. The BLA is the most important brain region of the circadian clock [47]. Thus, we propose that CDK5 dysfunction affected microtubule equilibrium and transport between the DS and the other brain structures associated with sleep-wake rhythms.

In the current study, we did not examine whether dysfunction of CDK5 is involved in nighttime sleep disturbances in animal models of PD, and this remains to be addressed in future studies. However, in an animal model of PD, in which the neurotoxin, MPTP, is used to selectively induce neurodegeneration of DA neurons in the substantia nigra, higher levels of CDK5 activity are detected. Furthermore, there is increased CDK5-mediated phosphorylation of DARPP-32 at Thr75, which is accompanied by decreased DA-induced phosphorylation of DARPP-32 at Thr34. Accordingly, CDK5 activity is not only required for dendrite and spine maintenance, but also for normal striatal dopamine signaling. The current findings, therefore, constitute a foundation for extending our understanding of the role of CDK5 in the pathogenesis of sleep-wake rhythm disorders in PD.

Together, our findings demonstrate a pivotal role of CDK5 in inter-regional connectivity, neurotransmission and motor control in the striatum. Targeted knockout of the $C d k 5$ gene in the DS produced major changes in dendrite structure at most E/I synapses of MSNs in the striatum. These changes impact communication between the DS and other brain regions, resulting in the dysregulated initiation of sleep-wake rhythm behaviors (summarized in Fig. 8). Collectively, our findings show that CDK5 in the DS plays a key role in the maintenance of striatal neural circuits associated with motor and circadian rhythm functions. 


\section{Abbreviations}

PD Parkinson's disease; SN: substantia nigra

DS Dorsal striatum

CDK5 Cyclin-dependent kinase 5

DA Dopamine

GABA y-aminobutyric acid

DARPP-32 Dopamine- and cAMP-regulated phosphoprotein of $32 \mathrm{kDa}$

MPTP 1-methyl-4-phenyl-1,2,3,6-tetrahydropyridine

CRISPR/Cas9 Clustered Regularly Interspaced Short Palindromic Repeats -associated 9

LV Lentivirus

SgRNA Small guide RNA

KD Knock down

NC Negative control

MSN Medium spiny neuron

i.p. Intraperitoneal

ACSF Artificial cerebrospinal fluid

TTX Tetrodotoxin; CNQX: 6-cyano-7-nitroquinoxaline-2, 3-dione

L-AP5 L-(+)-2-amino-5-phosphonopentanoic acid

mEPSC miniature excitatory presynaptic currents

sIPSC spontaneous inhibitory presynaptic currents

SEM Standard error of the mean

MAP2 Microtubule associated protein 2

PSD-95 Postsynaptic density protein 95

M2 Secondary motor cortex 


\section{Declarations}

\section{Ethics approval and consent to participate}

All animal procedures were carried out in accordance with the National Institutes of Health Guide for the Care and Use of Laboratory Animals (NIH Publications No. 80-23), with the approval of the Animal Care and Use Committees of the Beijing Institute of Pharmacology and Toxicology.

\section{Consent for publication}

Not applicable.

\section{Availability of data and materials}

All data generated or analyzed during this study are included in this published article and its supplementary information files.

\section{Competing interests}

The authors declare no competing financial interests.

\section{Funding}

This work was supported by the National Megaproject of Science Research of China (2018ZX09301-006) and the National Integrated Drug Discovery Technology Platform Foundation of China (2012ZX09301003).

\section{Authors' contributions}

Liyun Wang and Yongan Wang conducted the study and wrote the manuscript; Hu Zhou collected data, performed statistical analysis, and prepared figures; Jingxin Zhang, Huaxiang Shi, Pengfei Li and Xin Sui performed experiments. All authors read and approved the final manuscript.

\section{Acknowledgements}


We thank Barry Patel, PhD, from Liwen Bianji (Edanz) (www.liwenbianji.cn/), for editing the English text of a draft of this manuscript.

\section{References}

1. Poewe W, Seppi K, Tanner CM, Halliday GM, Brundin P, Volkmann J, et al. Parkinson disease. Nat Rev Dis Primers. 2017;3:17013.

2. Gonzales KK, Smith Y. Cholinergic interneurons in the dorsal and ventral striatum: anatomical and functional considerations in normal and diseased conditions. Ann N Y Acad Sci. 2015;1349:1-45.

3. Plotkin JL, Goldberg JA. Thinking Outside the Box (and Arrow): Current Themes in Striatal Dysfunction in Movement Disorders. Neuroscientist. 2019;25(4):359-79.

4. Modugno N, Lena F, Di Biasio F, Cerrone G, Ruggieri S, Fornai F. A clinical overview of non-motor symptoms in Parkinson's Disease. Arch Ital Biol. 2013;151(4):148-68.

5. van Rooden SM, Visser M, Verbaan D, Marinus J, van Hilten JJ. Patterns of motor and non-motor features in Parkinson's disease. J Neurol Neurosurg Psychiatry. 2009;80(8):846-50.

6. Schrempf W, Brandt MD, Storch A, Reichmann H. Sleep disorders in Parkinson's disease. J Parkinsons Dis. 2014;4(2):211-21.

7. Videnovic A, Golombek D. Circadian and sleep disorders in Parkinson's disease. Exp Neurol. 2013;243:45-56.

8. Trotti LM, Bliwise DL. Treatment of the sleep disorders associated with Parkinson's disease. Neurotherapeutics. 2014;11(1):68-77.

9. Wisor JP, Nishino S, Sora I, Uhl GH, Mignot E, Edgar DM. Dopaminergic role in stimulant-induced wakefulness. J Neurosci. 2001;21(5):1787-94.

10. Qu WM, Huang ZL, Xu XH, Matsumoto N, Urade Y. Dopaminergic D1 and D2 receptors are essential for the arousal effect of modafinil. J Neurosci. 2008;28(34):8462-9.

11. Volkow ND, Fowler JS, Logan J, Alexoff D, Zhu W, Telang F, et al. Effects of modafinil on dopamine and dopamine transporters in the male human brain: clinical implications. JAMA. 2009;301(11):1148-54.

12. Do J, Kim Jl, Bakes J, Lee K, Kaang BK. Functional roles of neurotransmitters and neuromodulators in the dorsal striatum. Learn Mem. 2012;20(1):21-8.

13. Hernandez-Flores T, Hernandez-Gonzalez O, Perez-Ramirez MB, Lara-Gonzalez E, Arias-Garcia MA, Duhne $M$, et al. Modulation of direct pathway striatal projection neurons by muscarinic $M(4)$-type receptors. Neuropharmacology. 2015;89:232-44.

14. Svenningsson P, Nishi A, Fisone G, Girault JA, Nairn AC, Greengard P. DARPP-32: an integrator of neurotransmission. Annu Rev Pharmacol Toxicol. 2004;44:269-96.

15. Nishi A, Snyder GL, Greengard P. Bidirectional regulation of DARPP-32 phosphorylation by dopamine. J Neurosci. 1997;17(21):8147-55. 
16. Liu L, Huang Y, Huang Q, Zhao Z, Yu J, Wang L. Muscarinic acetylcholine M4 receptors play a critical role in oxotremorine-induced DARPP-32 phosphorylation at threonine 75 in isolated medium spiny neurons. Neuropharmacology. 2017;117:376-86.

17. Ferreras S, Fernandez G, Danelon V, Pisano MV, Masseroni L, Chapleau CA, et al. Cdk5 Is Essential for Amphetamine to Increase Dendritic Spine Density in Hippocampal Pyramidal Neurons. Front Cell Neurosci. 2017;11:372.

18. Li X, Zhou H, Yang P, Shi HX, Xiong Y, Nie ZY, et al. Cyclin-dependent Kinase 5 Regulates Cortical Neurotransmission and Neural Circuits Associated with Motor Control in the Secondary Motor Cortex in the Mouse. Neuroscience. 2020;438:9-24.

19. Smith PD, Crocker SJ, Jackson-Lewis V, Jordan-Sciutto KL, Hayley S, Mount MP, et al. Cyclindependent kinase 5 is a mediator of dopaminergic neuron loss in a mouse model of Parkinson's disease. Proc Natl Acad Sci U S A. 2003;100(23):13650-5.

20. Hayashi A, Matsunaga N, Okazaki H, Kakimoto K, Kimura $Y$, Azuma H, et al. A disruption mechanism of the molecular clock in a MPTP mouse model of Parkinson's disease. Neuromolecular Med. 2013;15(2):238-51.

21. Hyacinthe C, Barraud Q, Tison F, Bezard E, Ghorayeb I. D1 receptor agonist improves sleep-wake parameters in experimental parkinsonism. Neurobiol Dis. 2014;63:20-4.

22. Lauretti E, Di Meco A, Merali S, Pratico D. Circadian rhythm dysfunction: a novel environmental risk factor for Parkinson's disease. Mol Psychiatry. 2017;22(2):280-86.

23. Kovalzon VM, Ugrumov MV, Pronina TS, Dorokhov VB, Manolov Al, Dolgikh VV, et al. [Early Stages of Parkinson's Disease: Comparative Characteristics of Sleep-Wakefulness Cycle in Patients and Model Animals]. Fiziol Cheloveka. 2015;41(6):114-8.

24. Chon U, Vanselow DJ, Cheng KC, Kim Y. Enhanced and unified anatomical labeling for a common mouse brain atlas. Nat Commun. 2019;10(1):5067.

25. Brown RW, Perna MK, Noel DM, Whittemore JD, Lehmann J, Smith ML. Amphetamine locomotor sensitization and conditioned place preference in adolescent male and female rats neonatally treated with quinpirole. Behav Pharmacol. 2011;22(4):374-8.

26. Birdsong WT, Jongbloets BC, Engeln KA, Wang D, Scherrer G, Mao T. Synapse-specific opioid modulation of thalamo-cortico-striatal circuits. Elife. 2019;8.

27. Mao M, Nair A, Augustine GJ. A Novel Type of Neuron Within the Dorsal Striatum. Front Neural Circuits. 2019;13:32.

28. Su SC, Tsai LH. Cyclin-dependent kinases in brain development and disease. Annu Rev Cell Dev Biol. 2011;27:465-91.

29. Katz LC, Burkhalter A, Dreyer WD. Fluorescent latex microspheres as a retrograde neuronal marker for in vivo and in vitro studies of visual cortex. Nature. 1984;310(5977):498-500.

30. Shah K, Rossie S. Tale of the Good and the Bad Cdk5: Remodeling of the Actin Cytoskeleton in the Brain. Mol Neurobiol. 2018;55(4):3426-38. 
31. Bykhovskaia M. Synapsin regulation of vesicle organization and functional pools. Semin Cell Dev Biol. 2011;22(4):387-92.

32. Verstegen AM, Tagliatti E, Lignani G, Marte A, Stolero T, Atias M, et al. Phosphorylation of synapsin I by cyclin-dependent kinase -5 sets the ratio between the resting and recycling pools of synaptic vesicles at hippocampal synapses. J Neurosci. 2014;34(21):7266-80.

33. Chellappa SL, Gaggioni G, Ly JQ, Papachilleos S, Borsu C, Brzozowski A, et al. Circadian dynamics in measures of cortical excitation and inhibition balance. Sci Rep. 2016;6:33661.

34. Hernandez A, Tan C, Mettlach G, Pozo K, Plattner F, Bibb JA. Cdk5 Modulates Long-Term Synaptic Plasticity and Motor Learning in Dorsolateral Striatum. Sci Rep. 2016;6:29812.

35. Hirono M, Yanagawa Y. Endocannabinoids regulate cerebellar GABAergic transmission in a synapse type-dependent manner. J Neurosci Res. 2021;99(3):898-913.

36. Borgkvist A, Avegno EM, Wong MY, Kheirbek MA, Sonders MS, Hen R, et al. Loss of Striatonigral GABAergic Presynaptic Inhibition Enables Motor Sensitization in Parkinsonian Mice. Neuron. 2015;87(5):976-88.

37. Uzunova G, Pallanti S, Hollander E. Excitatory/inhibitory imbalance in autism spectrum disorders: Implications for interventions and therapeutics. World J Biol Psychiatry. 2016;17(3):174-86.

38. Rabenstein M, Murr N, Hermann A, Rolfs A, Frech MJ. Alteration of GABAergic Input Precedes Neurodegeneration of Cerebellar Purkinje Cells of NPC1-Deficient Mice. Int J Mol Sci. 2019;20(24).

39. Dubbioso R, Ruggiero L, Esposito M, Tarantino P, De Angelis M, Aruta F, et al. Different cortical excitability profiles in hereditary brain iron and copper accumulation. Neurol Sci. 2020;41(3):679-85.

40. Mishra AK, Dixit A. Dopaminergic Axons: Key Recitalists in Parkinson's Disease. Neurochem Res. 2021.

41. Palumbo A, Gruning P, Landt SK, Heckmann LE, Bartram L, Pabst A, et al. Deep Learning to Decipher the Progression and Morphology of Axonal Degeneration. Cells. 2021;10(10).

42. Sheng ZH. Mitochondrial trafficking and anchoring in neurons: New insight and implications. J Cell Biol. 2014;204(7):1087-98.

43. Perrone M, Caroccia N, Genovese I, Missiroli S, Modesti L, Pedriali G, et al. The role of mitochondriaassociated membranes in cellular homeostasis and diseases. Int Rev Cell Mol Biol. 2020;350:11996.

44. Murakami M, Vicente MI, Costa GM, Mainen ZF. Neural antecedents of self-initiated actions in secondary motor cortex. Nat Neurosci. 2014;17(11):1574-82.

45. Dafsari HS, Silverdale M, Strack M, Rizos A, Ashkan K, Mahlstedt P, et al. Nonmotor symptoms evolution during 24 months of bilateral subthalamic stimulation in Parkinson's disease. Mov Disord. 2018;33(3):421-30.

46. Kurcova S, Bardon J, Vastik M, Vecerkova M, Frolova M, Hvizdosova L, et al. Bilateral subthalamic deep brain stimulation initial impact on nonmotor and motor symptoms in Parkinson's disease: An open prospective single institution study. Medicine. 2018;97(5):e9750. 
47. Wang Y, Liu Z, Cai L, Guo R, Dong Y, Huang YH. A Critical Role of Basolateral Amygdala-to-Nucleus Accumbens Projection in Sleep Regulation of Reward Seeking. Biol Psychiatry. 2020;87(11):954-66.

\section{Figures}

\section{Figure 1}

Lentivirus expressing Cdk5-sgRNA reduces CDK5 protein levels in the DS. Immunoassays were performed 14 days after LV/Cas9-sgRNA microinjection. (A) Schematic of the LV/Cas9-sgRNA expression vector. (B) Experimental design and schematic of CDK5 expression in the dorsal striatum (DS). Scale bar $=500 \mu \mathrm{m}$.

(C) Representative immunofluorescence image of CDK5 staining in the DS in CDK5-NC and CDK5-KD mice. Scale bar $=500 \mu \mathrm{m}$. (D) Representative western blot assay of CDK5 expression in the DS. Band intensity of CDK5 relative to $\beta$-actin is shown as the mean $\pm S E M, n=4$ independent experiments. Oneway ANOVA, Tukey's multiple comparisons test, ${ }^{\star} p<0.05,{ }^{\star \star} p<0.01,{ }^{\star \star \star} p<0.001$ vs WT.

\section{Figure 2}

DS-specific CDK5-KD affects motor activity of mice. (A) The locomotor activity test was performed at 14 days after CDK5 or NC-sgRNA microinjection. Movement was recorded for $30 \mathrm{~min}$ and is reported as distance in centimeters $(\mathrm{cm})$ per $5 \mathrm{~min}$. (B-C) ANOVA analysis of total distance travelled (B) and averaged speed (C) in the locomotor test of WT, DS-CDK5-NC and DS-CDK5-KD mice. (D) Activity heatmap of mice in the locomotor test. Blue indicates low activity and red indicates high activity. (E) Statistical analysis of the central distance traveled in the locomotor test chamber. $(F)$ The graph shows WT and DS-CDK5-NC mice exhibited normal activity with free-walking, as in the left panel, while the DSCDK5-KD mice remained immobile and curled up, as in the right panel during the test period. (G) Quantitative analysis of the curling up behavior in the locomotor test chamber. Data are represented as the mean $\pm \mathrm{SEM}, \mathrm{n}=8$ mice per group. One-way ANOVA, Tukey's multiple comparisons test, ${ }^{\star} p<0.05$, ${ }^{\star *} p$ $<0.01,{ }^{\star \star \star} p<0.001$ vs WT.

\section{Figure 3}

Altered circadian wheel-running activity correlates with loss of CDK5 in the DS. (A) Representative actograms showing wheel running activity during entrainment in a 12:12 night/day cycle, in which gray shading represents times of darkness. (B-C) Activity counts (B) and activity durations (C) in a 12:12 night/day cycle. DS-CDK5-KD mice displayed significant lower circadian free-running activity in darkness. (D) The ratios of 12-h night (a) to 12-h day ( $\rho$ ) activity durations of WT, DS-CDK5-NC and DS-CDK5-KD 
mice. (E) Pearson correlation between curling up duration in the locomotor activity test with the $a / \rho$ in circadian wheel-running test. Values " $R$ " and " $P$ " are indicated next to linear regression lines. Dashed curves indicate $95 \%$ confidence intervals. Data are represented as the mean $\pm S E M, n=8$ mice per group.

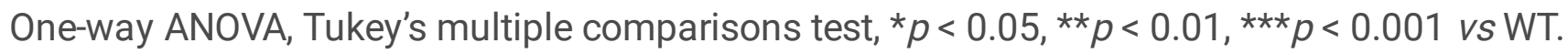

\section{Figure 4}

Effects of CDK5 knockdown on dendrite length and spine density of DS neurons. (A) Upper panel: Representative Golgi staining of dendrites in the DS of WT, DS-CDK5-NC and DS-CDK5-KD mice; Lower panel: 8-bit neuron tracing images. Scale bar $=20 \mu \mathrm{m}$. (B-D) Quantification of dendrite length and branching of MSN tracing images. (E) Representative photographs of MSN dendrite segments from the DS of WT, DS-CDK5-NC and DS-CDK5-KD mice. (F) Cell counter for spine density analysis. $n=10-20$ MSNs/segments from four slices of two animals per group. ANOVA analysis showing that there is a reduction in dendrite length, branching and spine density in DS-CDK5-KD mice compared with WT and DS-CDK5-NC mice. Data are represented as the mean \pm SEM. One-way ANOVA, Tukey's multiple comparisons test, ${ }^{*} p<0.05,{ }^{* *} p<0.01,{ }^{* \star *} p<0.001$ vs WT.

\section{Figure 5}

CDK5 deficiency reduces the expression of MAP2, synapsin 1, PSD95 and Tau phosphorylation in the DS. (A) Representative blots of MAP2, synapsin 1 and PSD95 from the DS of WT, DS-CDK5-NC, and DS-CDK5KD mice. (B) ANOVA analysis of relative protein levels of MAP2, synapsin 1 and PSD95. (C) Representative blots of total Tau, and phosphorylated Tau at Thr181 and Ser202 from the DS of WT, DSCDK5-NC, and DS-CDK5-KD mice. (D) ANOVA of relative Tau, p-Tau Thr181 and Ser202 levels. Data are represented as the mean \pm SEM, $n=3-4$ independent experiments. One-way ANOVA, Tukey's multiple comparisons test. ${ }^{\star} p<0.05,{ }^{\star \star} p<0.01,{ }^{\star \star \star} p<0.001$ vs WT.

\section{Figure 6}

CDK5 knockdown perturbs synaptic transmission in the DS. (A) Schematic depiction of electrode placements and action potential properties recorded from MSNs in the DS. (B) Representative mEPSC traces recorded from MSNs from WT, DS-CDK5-NC and DS-CDK5-KD mice. Scale bars $=20$ pA/200 ms.

(C) Cumulative histograms and quantification of mEPSC frequency and amplitude from (B). (D) Representative sIPSC traces recorded from MSNs of WT, DS-CDK5-NC and DS-CDK5-KD mice. Scale bars $=20 \mathrm{pA} / 400 \mathrm{~ms}$. (E) Cumulative histograms and quantification of mEPSC frequency and amplitude from 
(D). Data are represented as the mean $\pm S E M, n=12 \mathrm{MSN}$ from four mice per group. One-way ANOVA, Tukey's multiple comparisons test. ${ }^{\star} p<0.05,{ }^{\star \star} p<0.01,{ }^{\star \star \star} p<0.001$ vs WT.

\section{Figure 7}

CDK5 deficiency reduces long range projection from the DS to M2, Thalamic nucleus and BLA. (A) Experimental procedure. On the 14th day after LV/Cas9-sgRNA injection, AAV-hSyn-mCherry (red) was unilaterally injected into the DS. (B) Coronal section showing anterograde labeling 30 days after AAV injection (scale bar $=100 \mu \mathrm{m}$ ); the enlarged image shows multiple mCherry-labeled neurons in the DS (scale bar $=10 \mu \mathrm{m}) .(\mathrm{C}-\mathrm{E}) \mathrm{M} 2(\mathrm{C})$, Thalamic nucleus $(\mathrm{D})$ and BLA $(\mathrm{E})$ areas are labeled with mCherry projected from the DS. Data are represented as the mean $\pm S E M, n=3$ sections from three mice per group. One-way ANOVA, Tukey's multiple comparisons test. ${ }^{\star} p<0.05,{ }^{\star \star} p<0.01$, ${ }^{\star \star \star} p<0.001$ vs WT.

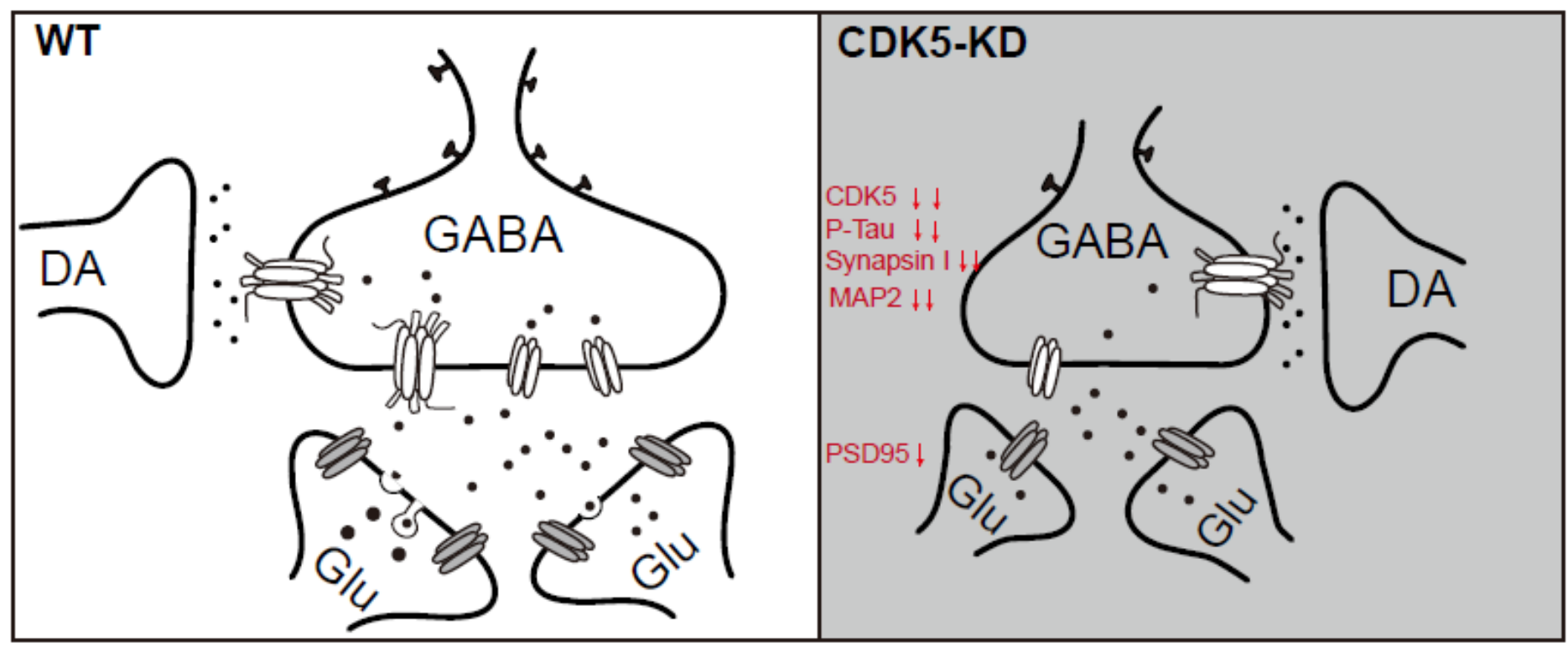

Normal dendritic morphology and synaptic transimission

Abnormal dendritic morphology and synaptic transimission

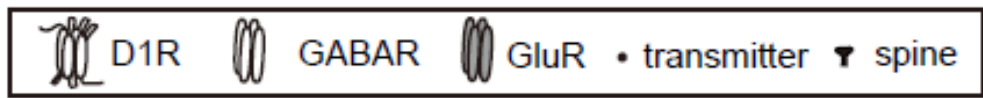

\section{Figure 8}

Proposed consequences of CDK5 loss on DS neuronal transmission. Compared with WT mice, shown on the left side, CDK5 deletion may cause a reduction in dendrite length and synapse density associated with downregulation of MAP2, PSD95, synapsin I and p-Tau Thr181 in DS. This results in impaired neuronal signaling and reduced long-range projections from the DS to $M 2$, Tha, and BLA. 